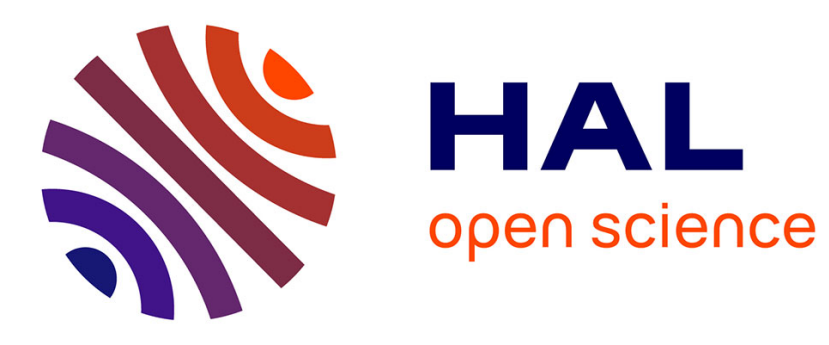

\title{
Caractérisation expérimentale du bruit au décollage du lanceur Ariane 5
}

M. Schott, B. Troclet, S. Vanpeperstraete

\section{To cite this version:}

M. Schott, B. Troclet, S. Vanpeperstraete. Caractérisation expérimentale du bruit au décollage du lanceur Ariane 5. Journal de Physique IV Proceedings, 1994, 04 (C5), pp.C5-977-C5-980. 10.1051/jp4:19945214 . jpa-00252899

\section{HAL Id: jpa-00252899 https://hal.science/jpa-00252899}

Submitted on 1 Jan 1994

HAL is a multi-disciplinary open access archive for the deposit and dissemination of scientific research documents, whether they are published or not. The documents may come from teaching and research institutions in France or abroad, or from public or private research centers.
L'archive ouverte pluridisciplinaire HAL, est destinée au dépôt et à la diffusion de documents scientifiques de niveau recherche, publiés ou non, émanant des établissements d'enseignement et de recherche français ou étrangers, des laboratoires publics ou privés. 


\title{
Caractérisation expérimentale du bruit au décollage du lanceur Ariane 5
}

\author{
M.O. SCHOTT, B. TROCLET ${ }^{*}$ et S. VANPEPERSTRAETE*
}

TEUCHOS Exploitation, 1 Avenue Foch, 78130 Les Mureaux, France

* Aérospatiale, BP. 2, 78133 Les Mureaux cedex, France

\begin{abstract}
At lift-off, a launch vehicle has to withstand a severe acoustic environment, induced by rocket jet and its impingement on the launch pad, which may damage the payloads and equipment. An experimental analysis of the lift-off noise applied to the ARIANE 5 launch vehicle was performed by ONERA in FAUGA MAUZAC facilities, in 1992 - 1993. This campaign, conducted on the responsibility of AEROSPATIALE as Industrial Architect within the framework of a CNES contract, intended to characterize the launcher acoustic environment and to test water injection as a noise reduction mean. This paper shows the 0.05 scale model launch vehicle and launch pad, equipped with 132 microphones in the frequency range $20-2000 \mathrm{~Hz}$ at full scale, the coherence between the different kinds of measurements, the research of analytical models for correlation functions, the test results in terms of levels versus altitude, correlation functions and the best mean of water injection. In conclusion, this campaign has given us all the data necessary to estimate the vibroacoustic response of the ARIANE 5 launch vehicle at lift-off in the frequency range of interest $20-2000 \mathrm{~Hz}$.
\end{abstract}

\section{INTRODUCTION}

Lors de son décollage, le lanceur est soumis à un champ de pression acoustique, généré par les jets des moteurs et par leur impact avec le pas de tir, qui excite les structures. Les charges induites dans la gamme de fréquence $20-2000 \mathrm{~Hz}$ peuvent endommager les charges utiles et les équipements.

C'est pourquoi, dès le début du programme Ariane 5 , il a été indispensable de caractériser le champ acoustique appliqué aux structures du lanceur et d'effectuer des calculs de réponse afin:

- d'estimer l'environnement acoustique des charges utiles et les niveaux de vibration aux points de fixation des équipements,

- de mettre en évidence un éventuel problème et, le cas échéant, de le résoudre.

Pour cela, le champ de pression acoustique, de nature aléatoire et large bande, doit être caractérisé au moyen des densités spectrales croisées.

Cet article présente les principaux résultats issus des essais de caractérisation du bruit au décollage, réalisés par l'O.N.E.R.A., sur une maquette rigide (échelle 1/20ème) du lanceur Ariane 5 et du pas de tir ELA3 muni de différents systèmes d'injection d'eau.

\section{DESCRIPTION DE L'ESSAI}

Un modèle échelle 1/20ème du lanceur Ariane 5 et de l'ensemble de lancement a été utilisé pour étudier le bruit au décollage du pas de tir. Cette campagne a été réalisée par l'O.N.E.R.A. sur le site du FAUGA à MAUZAC (Haute Garonne), sous la responsabilité de l'Architecte Industriel Ariane 5 AEROSPATIALE dans le cadre d'un contrat CNES. Les maquettes étaient équipées d'un total de 132 capteurs pour caractériser l'ambiance acoustique dans la gamme de fréquence $20-2000 \mathrm{~Hz}$ à l'échelle 1 , répartis de la façon suivante : 
- 36 capteurs pariétaux lanceur,

- 8 capteurs autour du moteur central,

- 46 capteurs champ libre disposés sur une antenne cylindrique autour de la Partie Haute du lanceur, ayant un diamètre double de celui du lanceur afin de s'affranchir des effets de diffraction,

- 18 capteurs sur les structures solides du pas de tir,

- 16 capteurs disposés sur une antenne linéaire près d'un propulseur,

- 8 capteurs disposés sur une antenne linéaire en sortie de carneau.

Un réseau fin de capteurs, centré sur un capteur en paroi de la coiffe, est utilisé pour déterminer les fonctions de corrélation.

Les figures 1 et 2 présentent l'installation d'essai et la position des capteurs pariétaux. La maquette lanceur est fixée sous un portique permettant de simuler des élévations du lanceur de 0 à $100 \mathrm{~m}$.

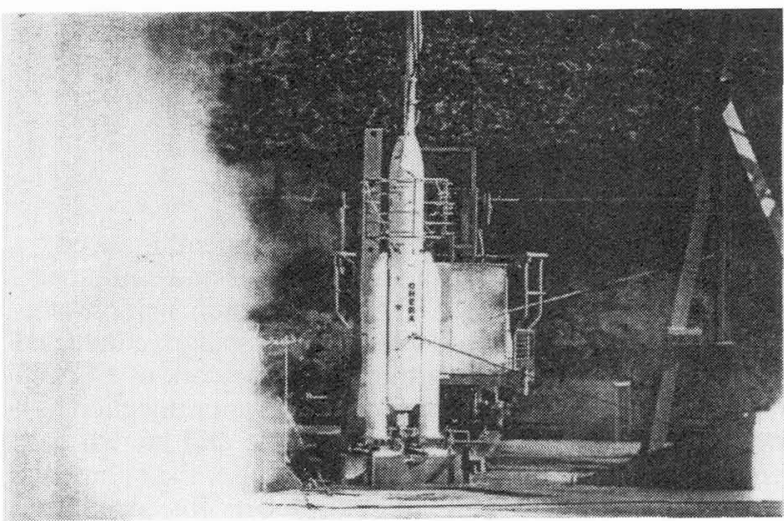

Photo de l'installation

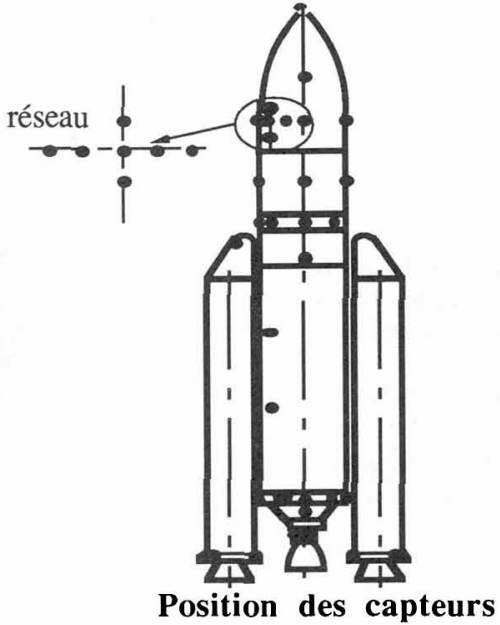

Figure 2

Figure 1

Vingt tirs ont été réalisés, principalement afin d'étudier l'évolution du bruit avec l'altitude du lanceur et de tester différents types d'injection d'eau : dans les carneaux pour modifier les propriétés thermodynamiques du jet dans lequel l'eau est injectée et sur la table de lancement pour créer une nappe d'eau afin d'amortir l'interaction jet / table. Chaque tir, effectué à une altitude du lanceur fixe, se décompose dans le temps par :

-l'allumage du moteur central, puis des deux Etages d'Accélération à Poudre,

- une première phase utile sans injection d'eau,

- une seconde phase utile avec injection d'eau.

Les quantités mesurées sont :

- les niveaux de pression RMS sur toute la durée de l'essai, soit 2 s. environ,

- les densités spectrales de puissance dans la bande 5-2000 Hz échelle 1 et les spectres par bande de tiers d'Octave de fréquences centrales 25 à $2000 \mathrm{~Hz}$ pour les phases avec et sans injection d'eau

- pour les capteurs montés en réseau : les autocorrélations, intercorrélations et densités spectrales croisées normalisées, ou fonctions de corrélation, pour les 2 phases.

\section{METHODE D'EXPLOITATION}

\subsection{Caractérisation du champ de pression externe}

Une détermination complète des densités spectrales croisées de la pression excitatrice nécessiterait un trop grand nombre de capteurs. Pour s'affranchir de cette difficulté matérielle, il est courant de faire les hypothèses suivantes:

- la densité spectrale de puissance de la pression est constante sur des surfaces finies de la structure,

- le champ de corrélation est statistiquement homogène sur une surface finie (sous-ensemble), c-à-d la fonction de corrélation entre les points $M$ et $M^{\prime}$ de la structure, définie comme suit :

$$
C\left(x, y, x^{\prime}, y^{\prime}, \omega\right)=\frac{S\left(x, y, x^{\prime}, y^{\prime}, \omega\right)}{\sqrt{S(x, y, \omega)^{*} S\left(x^{\prime}, y^{\prime}, \omega\right)}}
$$


ne dépend, outre la fréquence, que des différences $\left(x-x^{\prime}\right)$ et $\left(y-y^{\prime}\right)$. On désigne par $x, x^{\prime}$ et $y, y^{\prime}$ les coordonnées des points $M$ et $M^{\prime}$, par $S\left(x, y, x^{\prime}, y^{\prime}, \omega\right)$ la densité spectrale croisée entre les points $M$ et $M^{\prime}$ et par $S(x, y, \omega)$ la densité spectrale de puissance en $M$.

Physiquement, la fonction de corrélation intervient dans l'efficacité du bruit en terme de réponse structurale.

\subsection{Cohérence entre les différents types de mesures}

Lors de cette campagne d'essais, deux types de mesures acoustiques ont été effectuées:

- mesures pariétales, utilisées couramment,

- mesures en champ libre à l'aide d'une antenne cylindrique autour de la Partie Haute et utilisées pour la première fois dans le cadre du programme ARIANE 5.

Les niveaux de bruit et les fonctions de corrélation ont permis de vérifier la cohérence entre ces mesures. Les fonctions de corrélation mesurées en paroi du lanceur restent les mêmes en champ libre (sur l'antenne). La comparaison des niveaux mesurés en champ libre et en paroi a permis de dégager un effet de paroi, qui varie de 1 à $3 \mathrm{~dB}$ selon la bande de fréquence considérée, conformément aux prédictions effectuées sur un cylindre infini.

\section{RESULTATS OBTENUS}

Les résultats mesurés sur maquette sont transposés à l'échelle grandeur en divisant les fréquences par 20 , les pressions mesurées étant conservées.

\subsection{Niveau de bruit}

A partir des niveaux mesurés par chaque capteur, on déduit les niveaux de bruit moyens par octave appliqués à chaque sous-ensemble du lanceur. La variation de ces niveaux avec l'altitude du lanceur dépend de la position du sous-ensemble :

- Sur la partie haute, le niveau maximal est obtenu à l'altitude $50 \mathrm{~m}$ dans le domaine des basses et moyennes fréquences (jusqu'à $500 \mathrm{~Hz}$ ) et à $5 \mathrm{~m}$ dans le domaine des hautes fréquences.

- Sur la partie basse, le niveau maximal est obtenu à $5 \mathrm{~m}$ du sol, quelque soit la fréquence considérée. Les injections d'eau mises en place diminuent les niveaux de bruit sur le lanceur, d'autant plus que l'altitude de celui-ci est faible. Le système d'injection le plus efficace comprend :

- une injection dans le déflecteur du moteur central, qui permet de réduire notablement le bruit lorsque le lanceur est à $0 \mathrm{~m}$ du sol, également,

- une injection dans les carneaux des deux propulseurs EAP, pour réduire le bruit à faible altitude

- une injection sur la table de lancement, telle que les jets d'eau atteignent le plan supérieur des palettes EAP où viennent s'écraser les jets des moteurs. Cette injection est surtout efficace pour atténuer le bruit à l'altitude $50 \mathrm{~m}$, sur la partie haute du lanceur dans les premières bandes d'octave, ce qui est important vis à vis des charges utiles.

La figure 3 montre l'allure typique des spectres de bruit obtenus sur la partie haute du lanceur.

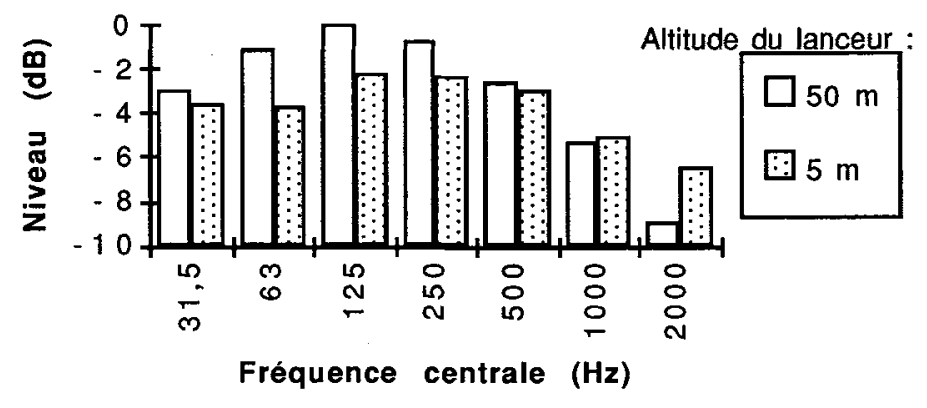

Spectre en partie haute du lanceur

Figure 3 


\subsection{Fonction de corrélation}

Pour les calculs de réponse vibroacoustique, les fonctions de corrélation sont définies analytiquement. L'expression de ces fonctions est basée sur les modèles suivant:

- axe longitudinal :

- axe circonférenciel :

$$
\mathrm{C}_{\mathrm{L}}(\xi, \omega)=\mathrm{A} \exp \left(-\frac{\mathrm{c}_{\mathrm{x}} \omega \xi}{\mathrm{a}_{\mathrm{x}} \mathrm{c}}\right) \cos \left(\frac{\mathrm{c}_{\mathrm{x}} \omega \xi}{\mathrm{c}}\right)+\mathrm{B} \operatorname{sinc}\left(\frac{\omega \xi}{\mathrm{c}}\right)
$$

$$
C_{T}(\eta, \omega)=A \exp \left(-\frac{c_{y} \omega \eta}{a_{y} c}\right) \cos \left(\frac{c_{y} \omega \eta}{c}\right)+B \operatorname{sinc}\left(\frac{\omega \eta}{c}\right)
$$

Les paramètres $\xi$ et $\eta$ sont définis de la façon suivante :

$$
\begin{aligned}
\xi & =|x-x| \\
\eta & =|y-y|
\end{aligned}
$$

$$
M(x, y) \text { et } M^{\prime}\left(x^{\prime}, y^{\prime}\right) \text { points sur la surface du lanceur }
$$

x désigne l'abscisse du point sur l'axe du lanceur et y son ordonnée selon la circonférence du lanceur.

Les coefficients $A, B, a_{x}, c_{x}, a_{y}$ et $c_{y}$ sont des constantes qui ont été déterminées par l'exploitation des essais en fonction de l'altitude. Á et $\mathrm{B}$ sont des pondérations appliquées respectivement au bruit de jet et au bruit diffus, $\mathrm{a}_{\mathrm{x}}$ et $\mathrm{a}_{\mathrm{y}}$ sont des facteurs d'amortissement tandis que $\mathrm{c}_{\mathrm{x}}$ et $\mathrm{c}_{\mathrm{y}}$ corrigent la vitesse du son.

Les fonctions obtenues à l'altitude de bruit maximum sont présentées en figures 4 et 5 pour des points distants de $1 \mathrm{~m}$ à l'échelle 1 . Elles restent valables avec ou sans système d'injection d'eau

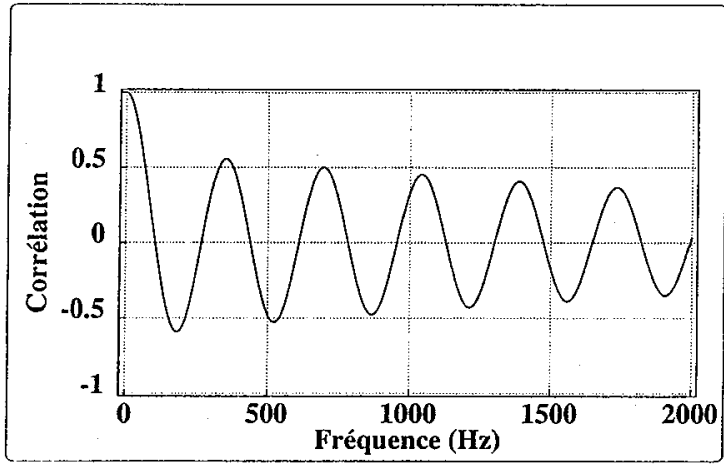

Corrélation longitudinale

Figure 4

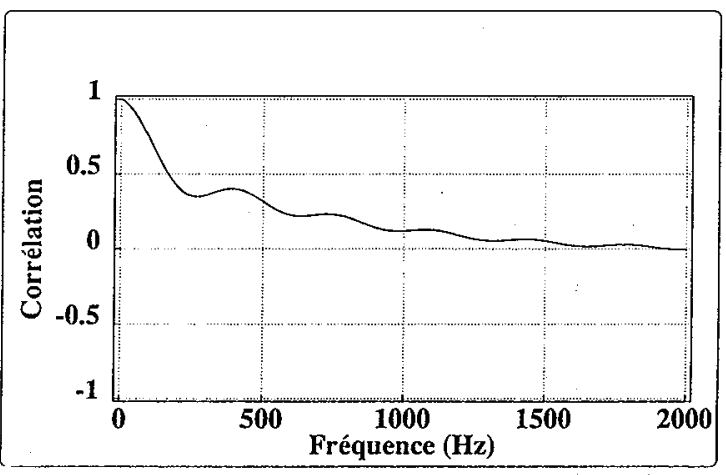

Corrélation transversale

Figure 5

\section{CONCLUSION}

L'expérience menée sur une maquette à l'échelle 1/20ème d'Ariane 5 a permis de définir le bruit appliqué au lanceur pendant son décollage, en termes de niveaux (densités spectrales de puissance) et de nature du bruit (fonctions de corrélation). Ces données servent d'entrée au calcul de réponse vibroacoustique du lanceur au bruit.

\section{REFERENCES}

[1] R.H. Lyon, Random Noise and Vibration in Space Vehicles, Library of Congress Catalog Card $\mathrm{N}^{\circ}$ 67-62274

[2] J. Lacaze, "Quelques questions générales concernant les vibrations des lanceurs - Vibrations haute fréquence", Scientific and Technical Review 1975 (1) 35-66.

[3] B. Troclet, T. Dang Trong, "Analysis of the Vibroacoustic Environment of Launchers", International Noise and Vibration Control Conference,St Petersburg, Russia, May 31-June 3, 1993. 